\title{
Validation of Whole-Slide Imaging for Histolopathogical Diagnosis: Current State
}

\author{
Adela Saco $^{\mathrm{a}} \quad$ Jose Ramírez ${ }^{\mathrm{a}}$ Natalia Rakislova ${ }^{\mathrm{a}} \quad$ Aurea Mira $^{\mathrm{a}} \quad \mathrm{Jaume} \mathrm{Ordi}^{\mathrm{a}}$ b

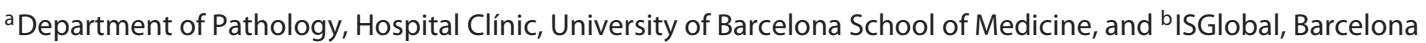 \\ Center for International Health Research (CRESIB), Barcelona, Spain
}

\section{Key Words}

Primary diagnosis $\cdot$ Routine diagnosis - Validation $\cdot$ Virtual microscopy $\cdot$ Whole-slide images

\begin{abstract}
Rapid advances in informatics and technological improvements have led to the development of high-throughput whole-slide imaging (WSI) scanners able to produce highquality digital images, which allow achieving a correct diagnosis of the biopsies using virtual viewers. This technology is currently prepared to be introduced in the departments of pathology for routine diagnosis. The aim of this review is to analyze the current evidence regarding the use of WSI in primary or routine diagnosis in the different subspecialties of pathology. An increasing number of studies have shown almost perfect inter- and intraobserver agreement between the diagnoses obtained with WSI and the classical diagnoses based on conventional light microscopy. The only exception seems to be cytology, which still requires some technological development. Although validation studies are needed in some areas of pathology, growing evidence indicates that WSI is a reliable tool for routine diagnosis. Pathologists have a positive perception of the ergonomics of the workstations, the low magnification of WSI and the possibility of making
\end{abstract}

annotations and measurements. WSI can be used from any device and anywhere, thereby providing great opportunities for teleconsultation. New technologies such as the recognition of histopathology patterns using image analysis may facilitate diagnosis and improve the reproducibility among pathologists in the future.

(c) 2016 S. Karger AG, Basel

\section{Introduction and Historical Perspective}

For more than a century, conventional light microscopy (CLM) has been the basic tool for tissue evaluation and has played a pivotal role in pathological diagnosis. Until the incorporation of nonmorphological molecular technologies into routine practice in recent years, the standard of diagnosis for pathologists was morphology and especially CLM-evaluated morphological criteria. Indeed, the evaluation of most specimens submitted to pathology laboratories today still relies on the interpretation of images by CLM, complemented by gross examination and a number of ancillary molecular techniques, most of which [histochemistry and immunohistochemistry (IHC)] are also evaluated with CLM. Asking experts or other colleagues for diagnostic opinions required sending

\section{KARGER}

E-Mail karger@karger.com www.karger.com/pat
(C) 2016 S. Karger AG, Basel

$1015-2008 / 16 / 0833-0089 \$ 39.50 / 0$
Jaume Ordi

Department of Pathology, Hospital Clínic, University of Barcelona C/Villarroel 170

ES-08036 Barcelona (Spain)

E-Mail jordi@clinic.ub.es 


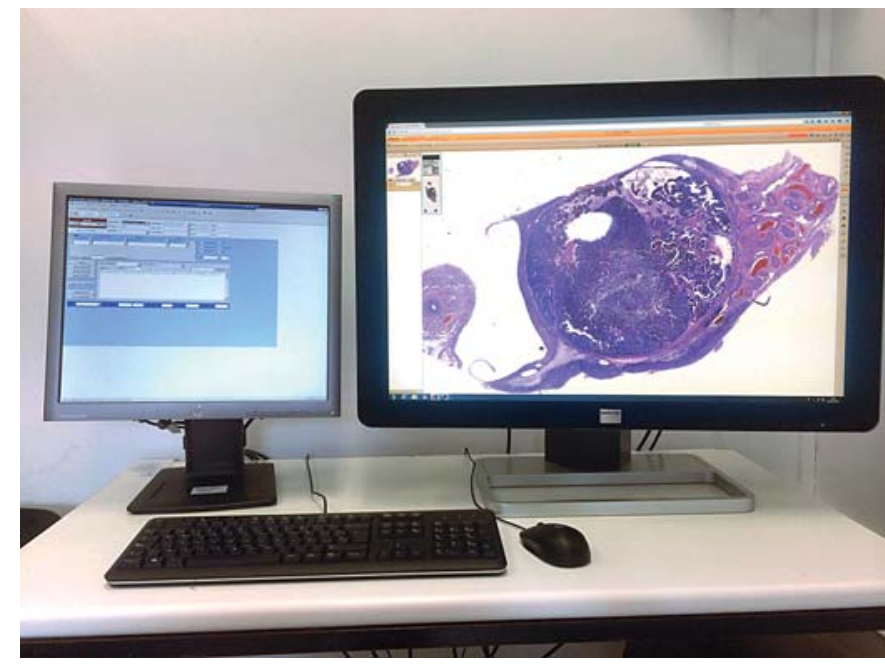

Fig. 1. WSI workstations for primary diagnosis typically include two screens, one displaying the WSI viewer and the other the laboratory information system and the clinical records or other clinical or imaging information. This physical structure has shown to be highly ergonomic. Additional advantages of WSI viewers are a much larger field of vision than CLM and the possibility of using a very low magnification.

glass slides or paraffin blocks for examination by CLM. Teaching pathology to undergraduates and residents, and continuing medical education for certified pathologists also depended on the use of CLM.

This scenario slowly started to change a few decades ago [1-3]. Static digital images allowed teaching and, to a certain degree, teleconsultation, but limitations in image quality and, particularly, the inability to navigate and use different optical objectives made the substitution of CLM unfeasible [4]. Dynamic real-time telepathology systems with video cameras integrated into CLM were used for intraoperative frozen biopsies, because they allowed an image to be sent to an expert located remotely. This capacity was extraordinarily useful for small hospitals, as it provided a quick diagnostic approach for difficult cases [5-12]. However, the relatively poor image quality and the impossibility to remotely conduct navigation through a slide made the system inadequate for routine diagnosis.

Rapid advances in informatics as well as technological improvements led to the development of scanners able to create digital reproductions from whole glass slides, which appeared one decade ago $[1,2]$. These scanners are the basis of virtual microscopy or whole-slide imaging (WSI), which allows navigation across the virtual slide and visualization at different magnifications, allowing the computer to be used as a CLM. However, the image qual- ity of the initial scanners was limited, and the costs of implementation of the technology, including the scanner, monitors and suitable computers, were very high, thereby restricting the use of WSI to certain areas, such as teaching and teleconsultation, and excluding routine diagnosis [10, 13-17].

Currently, a number of high-throughput scanners able to produce high-quality images are available on the market. These scanners allow correct diagnosis of the biopsies using virtual viewers. The cost of implementation of WSI has significantly decreased, and the speed of visualization has notably increased [17-22]. Constant improvements in this technology have led to an important expansion in the use of WSI in routine diagnosis in recent years. The aim of this review is to evaluate the current evidence on the validation of WSI in routine diagnosis.

\section{Advantages and Challenges of WSI for Routine Diagnosis}

Routine histopathological diagnosis can benefit from the multiple advantages of WSI. WSI workstations are more ergonomic (fig. 1). WSI has a much larger field of vision than CLM and allows a wider range of magnifications, thus providing easier navigation. In particular, WSI enables to study very low magnifications $(<\times 100)$, which is very useful in the evaluation of surgical specimens. The computer tools allow making annotations and measurements. WSI viewers can simultaneously show and synchronously move several slides of a case, which is particularly helpful in the evaluation of IHC-stained slides (fig. 2). Indeed, studies evaluating the opinion of pathologists have revealed a positive perception of image quality and stressed the utility of the measurement and annotation tools, as well as the ergonomics and usability of the viewer [22]. WSI can be used from any device and anywhere, thereby providing great opportunities for teleconsultation and remote work. Portability is certainly one of the major advantages of WSI, and this will probably be further improved in the near future when the current viewers are fully adapted to portable devices, such as tablets and smartphones [23-25]. Moreover, the need for standardization in the diagnosis and evaluation of IHC biomarkers predicting the outcome of specific therapies will probably boost the implementation of WSI.

Finally, WSIs allow for automatic quantification of IHC slides. These diagnostic algorithms facilitate quantification of IHC positivity resulting in a more objective evaluation, which is extremely useful in the evaluation of 
Fig. 2. WSI viewers may simultaneously show and synchronously move several slides of a case, which is particularly helpful in the evaluation of IHC-stained slides.

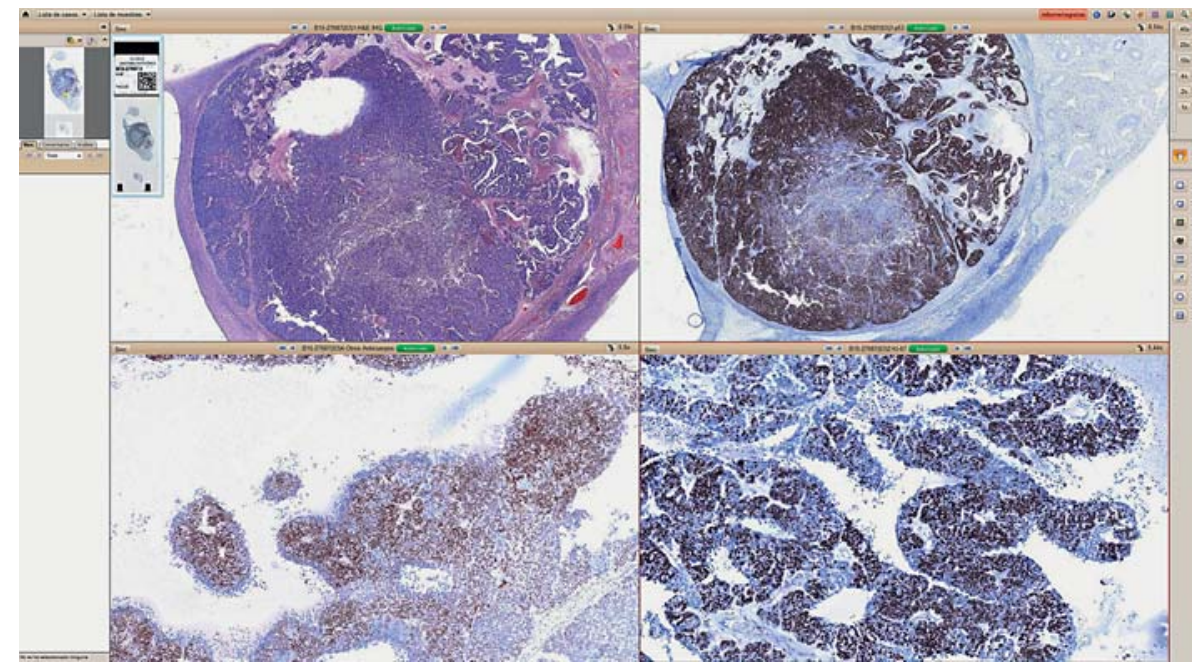

some biological markers. Algorithms for the evaluation of IHC stains are variably used depending on the subspecialties and are particularly useful in cases of breast cancer [26-29].

In contrast with these positive opinions, many pathologists still prefer using CLM. The most criticized feature of WSI is the speed in uploading the image. Indeed, most pathologists feel that more time is required to make a diagnosis with WSI. However, some studies have shown that although diagnosis with WSI is initially more timeconsuming, this time quickly decreases as pathologists become familiar with the use of the WSI viewer [30-35]. Thus, there is a learning curve in the use of WSI and the time required for making a diagnosis, and a recent study conducted at our institution confirmed that the diagnostic performance improved with practice [36]. Another limitation of WSI is the relatively high costs of the equipment. The basic needs for a WSI system, which is adequate for routine diagnosis, include not only highthroughput scanners but also high-resolution monitors $[37,38]$. This is a common concern since, despite the reduction in the price of the equipment in the last few years, it still represents a considerably high investment, which has a relatively low added value for many pathologists as the basic functions of WSI are already being confidently achieved with the old CLM. Finally, WSI requires a significant investment in high-capacity servers; the files generated by WSI scanners are huge, with sizes frequently over 2 GB per slide. Thus, strategies to reduce the size of the files, such as scanning at relatively low magnification $(\times 200$ instead of $\times 400$ or $\times 600)$ are frequently used [37].

Validation of WSI in Primary Diagnosis

\section{The Need for Validation Studies}

The number of studies aimed at validating WSI in primary or routine diagnosis is rapidly increasing. However, whereas relatively abundant information is available in some areas, validation studies are very scant in several subspecialties and completely absent in others. Some validation studies include biopsies from several subspecialties instead of analyzing biopsies with similar characteristics [33, 39-43]. This relative absence of validation studies has led to reluctance in the implementation of WSI in routine clinical practice. Nevertheless, the number of centers implementing this technology is increasing due to the positive experiences reported in many departments $[41,42,44,45]$.

Below, we review the current evidence on the validation of WSI versus CLM in the different subspecialties of pathology.

\section{Breast Pathology}

WSI has been validated in the diagnosis of breast pathology in a number of studies conducted by different groups. Most of these studies analyzed a relatively small number of routine biopsies (between 100 and 150), including either only needle biopsies or both needle and surgical specimens $[32,46,47]$. Although scanning at $\times 400$ was recommended in one of the studies [32], in two of the studies a scanning magnification of $\times 200$ was considered as sufficient $[46,47]$.

The intra- and interobserver agreement between CLM and WSI is excellent in all the studies, with values ranging 
Fig. 3. A major advantage of digitization in breast pathology is the possibility to use image analysis in improving the accuracy and reliability of HER-2, estrogen and progesterone receptors and Ki67 scoring, which have a crucial role in the planning of treatment strategies.

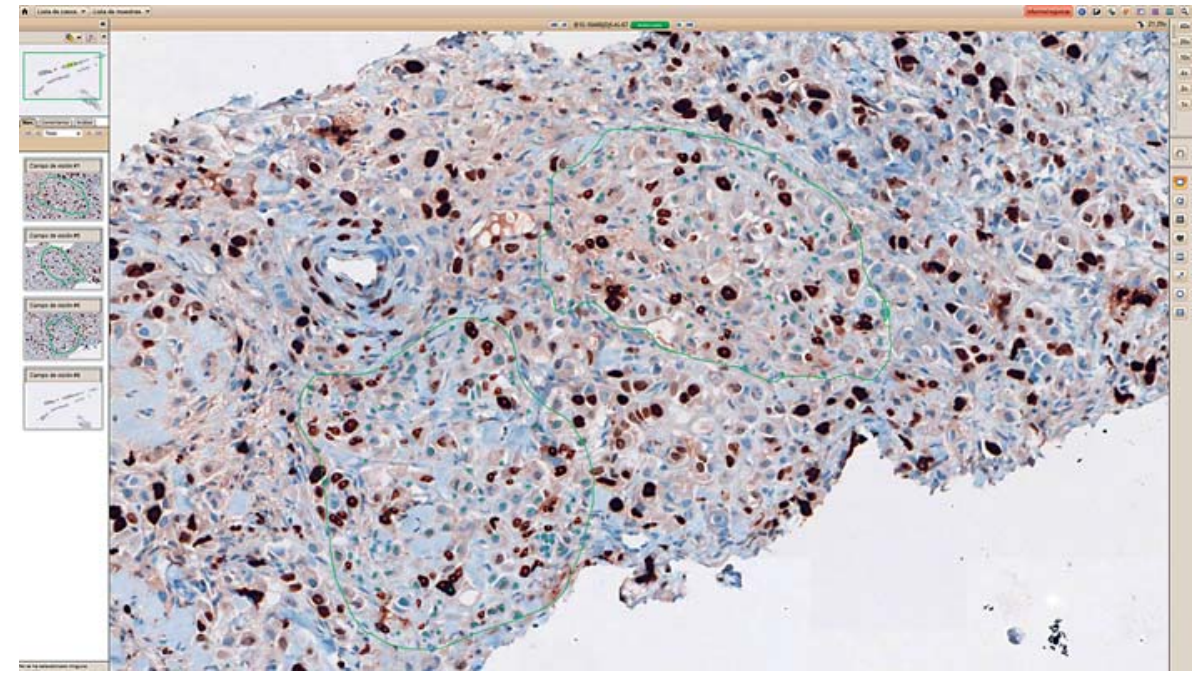

between 90 and 99\%. Most of the discrepancies detected did not have clinical repercussion. Interestingly, in two of the reports, the WSI diagnosis was more frequently considered as correct compared to the diagnosis performed with CLM $[32,46]$. A study specifically dealing with the distinction between hyperplasia and cancer reported interobserver concordance in the diagnosis of $90.2 \%$. Major discrepancies appeared in $2.3 \%$ of the cases, which, in most cases, were solved with IHC stains [48].

A major advantage of digitization in breast pathology is the possibility to use image analysis to improve the accuracy and reproducibility of HER-2, estrogen and progesterone receptors, and Ki67 scoring, which have a crucial role in the planning of treatment strategies [27-29, 49]. Moreover, the evaluation may be improved with the use of automatic quantification algorithms (fig. 3).

\section{Cytopathology}

The use of WSI in cytopathology has shown some advantages in second opinions, quality assurance, slide archiving, proficiency testing and education. However, a number of significant weaknesses of the current WSI scanners, such as the difficulties in focusing at different $\mathrm{z}$-axes, are a major limitation for the introduction of this technology in routine diagnosis $[50,51]$. Improvements in informatics may allow multiplane focusing using the $z$-axis, but they still need to be validated $[21,52,53]$.

Indeed, the current evidence of validation in cytology is almost limited to real-time dynamic digital microscopy using a video camera connected to the optical microscope and not to WSI. The intraobserver agreement of this approach with the final diagnosis is high (92\%) [54], and, in some studies, it is better than with CLM [53-55]. One study evaluating 192 liquid-based cervical cytology slides showed good intraobserver concordance (89-97\%), but the interobserver concordance was better for CLM than for WSI (94 vs. 82\%) [52].

\section{Dermatopathology}

Only two studies have focused on the validation of skin biopsies evaluating routine specimens. Although both studies included a small number of cases (100 and 79 , respectively), the intraobserver agreement was high (94\% for WSI and 96\% for CLM, respectively) [30, 56]. A study limited to tumor and tumor-like skin lesions showed agreement in the diagnosis by WSI and CLM, with a $\kappa$ value of 0.93 for both methods [57]. Another study evaluated inflammatory and melanocytic lesions, with good agreement between CLM and WSI (only 1 discordant diagnosis in the inflammatory biopsies and $100 \%$ concordance in the melanocytic specimens), but the number of patients included was very limited (24 cases). In this study, it was concluded that in most cases scanning at $\times 200$ is sufficient to achieve a correct diagnosis [56].

Interestingly, WSI has shown to be suitable for teleconsultation in skin biopsies and may reduce the time of response in expert diagnosis from 5-10 days to a few hours or even minutes [57]. 
Fig. 4. Prostatic biopsies often require multiple measurements. The tools of WSI viewers make these measurements easy. WSI allows a global view to more adequately establish the Gleason score.

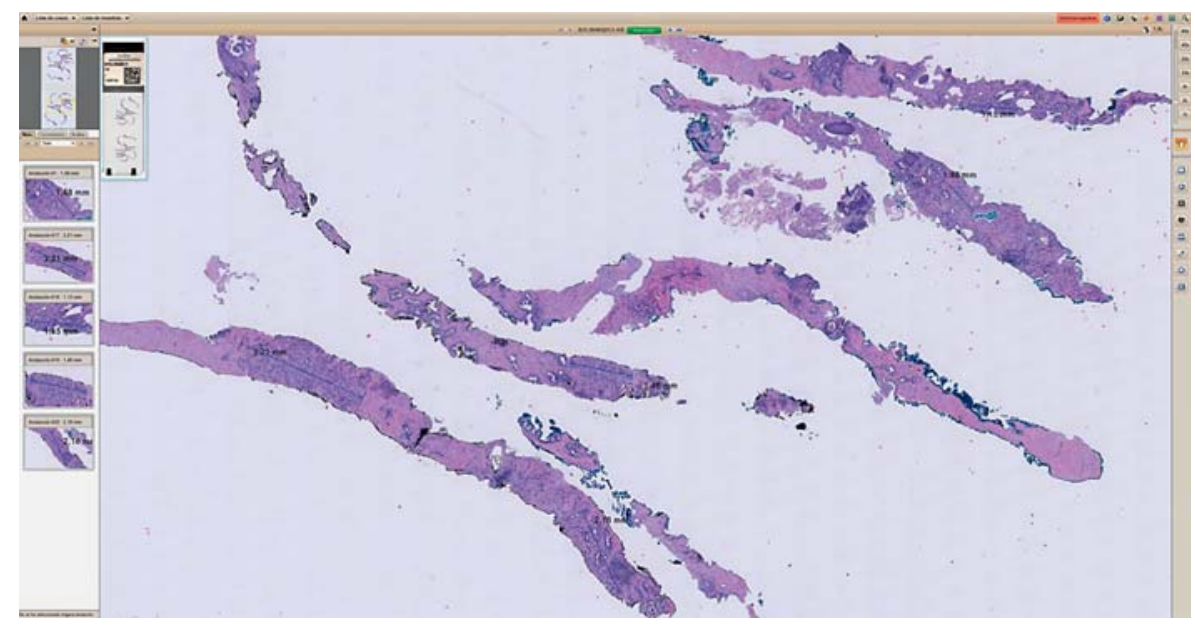

\section{Gastrointestinal Pathology}

A few studies have shown that the diagnosis of gastrointestinal biopsies using WSI or CLM provides comparable results $[58,59]$. Two reports analyzed consecutive routine biopsies, but one was limited to gastric and colonic biopsies [59]. The intraobserver concordance between WSI and CLM was 95\% in both studies, and scanning at $\times 200$ was considered as adequate. One study compared WSI and CLM in the evaluation of polyps in surgical specimens. Although the intra- and interobserver agreement was excellent for both methods in terms of diagnosis, WSI facilitated the quantification of the polyps due to the very low magnification that allows a panoramic view of the complete sample [60]. A study focused on Barrett's dysplasia and neoplasia showed good diagnostic agreement between WSI and CLM, but the consensus neoplasia score was lower using WSI and the time spent in making the diagnosis was longer. These results were probably due to the lack of confidence and experience in the manipulation of the WSI viewer and seemed to improve with familiarity and practice [34].

\section{Genitourinary Pathology}

Prostatic biopsies, particularly needle biopsies, are good candidates for digitization for a number of reasons: the tissue size is small and the images generated are lighter; multiple measurements are frequently required and informatics tools can facilitate these, and WSI allows a global view to more easily establish the Gleason score (fig. 4) [61]. An additional advantage of WSI is the pos- sibility to synchronize hematoxylin-eosin stains and p63 IHC in the same screen, thereby allowing the comparison of the two images and facilitating the diagnostic and teaching process [62].

Thus, the current evidence on the validation of WSI in the diagnosis of prostatic biopsies is more extensive than in other areas. A number of studies (from 50 to over 800 cases) have been focused on the evaluation of the Gleason score in needle biopsies. Scanning at $\times 200$ was considered sufficient to make the diagnosis. The $\kappa$ values for diagnosis ranged between 0.586 and 0.813 [63-65], and one of the reports included only difficult biopsies with a borderline Gleason score. Concordance between WSI and CLM seems to be higher for primary ( $\kappa$ values $0.65-0.96$ ) than for secondary Gleason scores ( $\kappa$ values $0.53-0.75$ ), and most discordances have no impact on patient management [66]. Tumor size is better evaluated with WSI, and other parameters such as perineural invasion show similar values with WSI and CLM [66].

Two additional studies focused on genitourinary biopsies included a mixture of prostatic and urinary tract biopsies and showed good intraobserver concordance (90 and $87.5 \%$, respectively) $[67,68]$.

\section{Gynecological Pathology}

Studies on the validation of WSI in gynecological biopsies are scant. Only one study conducted at our institution analyzed interobserver agreement in 452 routine gynecological specimens showing a $\kappa$ index of 0.914 (almost perfect concordance). Interestingly, the agreement between WSI and CLM increased in this study in parallel 
with time, suggesting that there is a learning curve in the use of WSI and that experience in the use of WSI viewers improves the results obtained. Major discrepancies were found in only $2 \%$ of the cases, and none was related to poor image quality. Most discrepancies in this study were observed in biopsies of premalignant lesions of the uterine cervix, an area which has shown high inter- and intraobserver variability rates using CLM [36]. The magnification used in the study was $\times 200$, and higher magnification did not seem to be required.

A second study described the usefulness of WSI in the evaluation of 52 frozen ovarian sections showing $96 \%$ interobserver agreement. Interestingly, in this study, no clinical information was provided to the pathologists, and the time spent per case was 3-5 min [7].

\section{Head and Neck Pathology}

To date, no validation study including the complete spectrum of samples of this subspecialty is available. Only one study on premalignant laryngeal lesions has been published. This study concluded that WSI is a valid alternative to CLM. Although the correlation with the final diagnosis was slightly lower with WSI than with CLM, the differences were not statistically significant [69].

\section{Neuropathology}

Validation studies of neuropathology are limited to intraoperative biopsies and smears [5, 8, 70]. Agreement between the diagnosis with WSI and the final diagnosis using CLM is very good, even with low scan magnification $(\times 100)$. The studies conclude that $\times 200$ magnification is sufficient to obtain a diagnosis. In one study, the diagnosis achieved with WSI was concordant with CLM in 29 of the 30 cases evaluated, and the discordant diagnosis did not lead to changes in the management of the patient [8]. A second study included 126 frozen sections that were evaluated by four different pathologists. The diagnosis was discordant with the final report in only 8 cases. In this study, the diagnosis of frozen sections scanned and diagnosed using WSI was compared with the final diagnosis obtained in formalin-fixed, paraffinembedded tissue [70].

Algorithms are currently being developed to identify the hot spots in Ki67-stained sections to automatically quantify the proliferative activity in tumors of the central nervous system $[71,72]$.

\section{Pediatric Pathology}

Two studies have validated the use of WSI in pediatric pathology. One included 80 routine biopsies of patients under 18 years of age and 20 placentas. The intraobserver concordance between the diagnoses with WSI and CLM was $90 \%$ in pediatric biopsies and $93 \%$ in placental specimens. Major discrepancies were observed in only $2 \%$ of the cases. A scanning magnification of $\times 200$ generated an image quality allowing correct diagnosis except for the identification of nucleated red blood cells, which is very difficult even when the slides are scanned at a magnification of $\times 400$ [73].

The second study evaluated WSI in 60 cases selected to include the whole spectrum of the diagnostic complexity of pediatric biopsies. The surgical specimens were digitized at $\times 200$ magnification, whereas small biopsies and cytological samples were digitized at $\times 400$. The intraobserver agreement was almost perfect with only 1 discordant case. The scanning process of two cytological smears was unsatisfactory because the material was very scanty [74].

\section{Pulmonary Pathology}

One study validating WSI in the diagnosis of intraoperative pulmonary specimens included a variety of samples, with 114 frozen sections from tumors, lymph nodes and bronchial margins, 174 fine-needle aspiration slides, 3 exfoliative smears and 13 small biopsies. This study evaluated both a dynamic real-time telepathology system and WSI, and found very good agreement, which was better for WSI than for the real-time telepathology system ( $100 \%$ in consultation and frozen biopsies) [75]. A second study analyzed the use of WSI in 20 tumor biopsies and surgical specimens sent for consultation. Complete interobserver agreement was achieved in $85 \%$ of the cases, even at a scanning magnification of $\times 100$ [75].

\section{Renal Pathology}

Validation studies of WSI in the diagnosis of renal pathology biopsies are scarce and include few cases. A report including 50 routine renal biopsies showed complete intraobserver agreement in $84 \%$ of the cases. Five major discrepancies (with clinical repercussion for the patient) were found and in 2 cases the correct diagnosis was made with WSI. In this study, renal transplant biopsies showed significantly more discrepancies at a magnification of 
$\times 200$ [67]. Another study (using a magnification of $\times 400$ ) reported good agreement in renal transplant biopsies, but the time spent for obtaining the diagnosis was longer with WSI than with CLM [76]. Finally, one study evaluated the concordance between 96 pathologists in the diagnosis of 12 renal biopsies using WSI and CLM and found no significant differences between both methods [77].

\section{Intraoperative Diagnosis of Frozen Sections}

A number of studies have evaluated dynamic real-time telepathology in intraoperative sections, showing a good correlation with CLM diagnosis. They emphasize the learning curve in the use of the WSI technology, which typically involves a longer diagnostic time window at the beginning but rapid improvement with practice [78]. A validation study using WSI in frozen intraoperative sections from different anatomical sites has shown almost perfect agreement with a $\kappa$ index of 0.85 . The mean time spent on diagnosis was 2 min 50 s per case. The quality of the image was considered excellent in $98 \%$ of cases [9]. Studies using WSI in frozen intraoperative sections from specific specialties have been discussed above. In another study evaluating 67 consecutive frozen intraoperative sections, diagnosis was obtained by viewing the virtual slides in a portable device (iPad tablet). The slides were scanned at $\times 200$, and all cases were shown together with the clinical information. The concordance between the diagnoses achieved with WSI and CLM was good with a $\kappa$ value of 0.85 . The mean time to achieving a diagnosis using WSI was 2 min and $46 \mathrm{~s}$ [79].

\section{Surgical Pathology}

A number of studies have evaluated a variety of different specimens from the routine practice in the department of pathology, including between 25 and 607 samples [22, 39-41, 80, 81]. Inter- and intraobserver agreement between WSI and CLM varied from 75 to $97.7 \%$ depending on the study. Most studies concluded that a magnification of $\times 200$ provides images with adequate quality for diagnosis $[22,39-41,80,81]$. The interobserver agreement between WSI and CLM was 95\%, and all discrepancies were minor. However, although the general opinion of the pathologists was positive, some felt that the WSI system was slower than CLM, and most of the pathologists interviewed were reluctant to completely move from CLM to WSI in routine diagnosis [33]. One study sug-

Validation of WSI in Primary Diagnosis gested that the interobserver agreement was better for neoplastic than for nonneoplastic diseases [16]. It has been suggested that a scanning magnification of $\times 200$ may not be sufficient to allow correct diagnosis in inflammatory lesions [82].

Finally, two studies included only consultation biopsies of different organs. The interobserver agreement between WSI and CLM diagnoses in these studies was greater than $91 \%$, and most of the discrepancies were due to the intrinsic difficulty in diagnosing some cases $[15,16,82]$.

\section{Current Recommendations for WSI Validation}

Validation of WSI at each institution has been recommended before its implementation in routine diagnosis. Several professional associations have developed guidelines with recommendations for the introduction of WSI in routine diagnosis in a department of pathology. The first guidelines were developed by the College of American Pathologists and the American Telemedicine Association, and include some recommendations and suggestions to be followed before using WSI for diagnosis $[2,10$, 17]. It is recommended to include a variety of different biopsies representative of the complexity of the surgical specimens usually analyzed in the center. The guidelines state that it is not necessary to validate each subspecialty because the results from one specialty can be extrapolated to others with similar features. Each specific type of specimen with significant differences requires an internal validation. The guidelines recommend measuring intraobserver agreement between WSI and CLM, using a 'washout period' of 2 weeks. Finally, it is recommended that a pathologist with experience in WSI should be involved in the process of validation.

\section{Conclusions}

In conclusion, independently of the subspecialty, all the validation studies published show a very good correlation between diagnoses achieved with WSI and CLM. Thus, WSI seems to be an adequate tool for histological diagnosis in routine practice and has several advantages over CLM. However, although good evidence demonstrating that WSI can be reliably used for routine diagnosis has been provided for several specialties, there are a number of areas of pathology, such as hematopathology and liver, endocrine, bone and soft-tissue pathology, for which no study has yet been published. Although some of 
these areas may be considered similar to others already validated, specific validation studies are needed in areas with many differences such as liver biopsies or hematopathology. These validations are necessary before the use of WSI can be extended to these subspecialties with the aim of going fully digital in pathological services in the future.

Notwithstanding, as with many other new tools, the use of WSI has a learning curve, and the time spent on the diagnosis and, to a lesser extent, inter- and intraobserver agreement may be suboptimal in the initial phases of its use. Cytology seems to be an exception; the application of WSI in this area is more controversial due to the impossibility of focusing on different planes.
However, the introduction of WSI in routine diagnosis faces some difficulties, mainly related to the reluctance of pathologists to abandon CLM and to the costs associated with the acquisition of the equipment and the storage of the images generated. New technologies that allow creating $3 \mathrm{D}$ reconstruction from $2 \mathrm{D}$ biopsies may help to improve the understanding of the growth patterns and the spatial arrangement of diseased cells [21, 83]. Another area that will markedly expand in the next few years is that of histopathology pattern recognition using image analysis, which can facilitate the diagnostic tasks and improve the reproducibility among pathologists in many subspecialties [65, 84-90].

\section{References}

1 Al-Janabi S, Huisman A, van Diest PJ: Digital pathology: current status and future perspectives. Histopathology 2012;61:1-9.

-2 Pantanowitz L, Sinard JH, Henricks WH, Fatheree LA, Carter AB, Contis L, Beckwith BA, Evans AJ, Lal A, Parwani AV: Validating whole slide imaging for diagnostic purposes in pathology: guideline from the College of American Pathologists Pathology and Laboratory Quality Center. Arch Pathol Lab Med 2013;137:1710-1722.

3 Weinstein RS: Prospects for telepathology. Hum Pathol 1986;17:433-434.

4 Cross SS, Dennis T, Start RD: Telepathology: current status and future prospects in diagnostic histopathology. Histopathology 2002; 41:91-109.

5 Evans AJ, Kiehl TR, Croul S: Frequently asked questions concerning the use of whole-slide imaging telepathology for neuropathology frozen sections. Semin Diagn Pathol 2010;27: 160-166.

-6 Evans AJ, Chetty R, Clarke BA, Croul S, Ghazarian DM, Kiehl TR, Ordonez BP, Ilaalagan S, Asa SL: Primary frozen section diagnosis by robotic microscopy and virtual slide telepathology: the University Health Network experience. Semin Diagn Pathol 2009;26:165-176.

7 Fallon MA, Wilbur DC, Prasad M: Ovarian frozen section diagnosis: use of whole-slide imaging shows excellent correlation between virtual slide and original interpretations in a large series of cases. Arch Pathol Lab Med 2010;134:1020-1023.

8 Gould PV, Saikali S: A comparison of digitized frozen section and smear preparations for intraoperative neurotelepathology. Anal Cell Pathol (Amst) 2012;35:85-91.

-9 Kaplan KJ, Burgess JR, Sandberg GD, Myers CP, Bigott TR, Greenspan RB: Use of robotic telepathology for frozen-section diagnosis: a retrospective trial of a telepathology system for intraoperative consultation. Mod Pathol 2002;15:1197-1204.
10 Pantanowitz L, Dickinson K, Evans AJ, Hassell LA, Henricks WH, Lennerz JK, Lowe A, Parwani AV, Riben M, Smith CD, Tuthill JM, Weinstein RS, Wilbur DC, Krupinski EA, Bernard J: American Telemedicine Association clinical guidelines for telepathology. J Pathol Inform 2014;5:39.

11 Slodkowska J, Pankowski J, Siemiatkowska K, Chyczewski L: Use of the virtual slide and the dynamic real-time telepathology systems for a consultation and the frozen section intraoperative diagnosis in thoracic/pulmonary pathology. Folia Histochem Cytobiol 2009; 47:679-684.

12 Wilbur DC: Digital pathology: get on board - the train is leaving the station. Cancer Cytopathol 2014;122:791-795.

13 Ayad E: Virtual telepathology in Egypt, applications of WSI in Cairo University. Diagn Pathol 2011;6(suppl 1):S1.

14 Romero LG, Cable W, Lesniak A, Tseytlin E, McHugh J, Parwani A, Pantanowitz L: Digital pathology consultations - a new era in digital imaging, challenges and practical applications. J Digit Imaging 2013;26:668-677.

$\checkmark 15$ Wienert S, Beil M, Saeger K, Hufnagl P, Schrader T: Integration and acceleration of virtual microscopy as the key to successful implementation into the routine diagnostic process. Diagn Pathol 2009;4:3.

16 Wilbur DC, Madi K, Colvin RB, Duncan LM, Faquin WC, Ferry JA, Frosch MP, Houser SL, Kradin RL, Lauwers GY, Louis DN, Mark EJ, Mino-Kenudson M, Misdraji J, Nielsen GP, Pitman MB, Rosenberg AE, Smith RN, Sohani AR, Stone JR, Tambouret RH, Wu CL, Young $\mathrm{RH}$, Zembowicz A, Klietmann W: Wholeslide imaging digital pathology as a platform for teleconsultation: a pilot study using paired subspecialist correlations. Arch Pathol Lab Med 2009;133:1949-1953.
17 Bernard C, Chandrakanth SA, Cornell IS, Dalton J, Evans A, Garcia BM, Godin C, Godlewski M, Jansen GH, Kabani A, Louahlia S, Manning L, Maung R, Moore L, Philley J, Slatnik J, Srigley J, Thibault A, Picard DD, Cracower H, Tetu B: Guidelines from the Canadian Association of Pathologists for establishing a telepathology service for anatomic pathology using whole-slide imaging. J Pathol Inform 2014;5:15.

18 Hedvat CV: Digital microscopy: past, present, and future. Arch Pathol Lab Med 2010;134: $1666-1670$

19 Ho J, Ahlers SM, Stratman C, Aridor O, Pantanowitz L, Fine JL, Kuzmishin JA, Montalto MC, Parwani AV: Can digital pathology result in cost savings? A financial projection for digital pathology implementation at a large integrated health care organization. J Pathol Inform 2014;5:33.

20 Isaacs M, Lennerz JK, Yates S, Clermont W, Rossi J, Pfeifer JD: Implementation of whole slide imaging in surgical pathology: a value added approach. J Pathol Inform 2011;2:39.

21 Pantanowitz L: Digital images and the future of digital pathology. J Pathol Inform 2010;1: 15.

22 Thorstenson S, Molin J, Lundstrom C: Implementation of large-scale routine diagnostics using whole slide imaging in Sweden: digital pathology experiences 2006-2013. J Pathol Inform 2014;5:14.

23 Hartman DJ, Parwani AV, Cable B, Cucoranu IC, McHugh JS, Kolowitz BJ, Yousem SA, Palat V, Reden AV, Sloka S, Lauro GR, Ahmed I, Pantanowitz L: Pocket pathologist: a mobile application for rapid diagnostic surgical pathology consultation. J Pathol Inform 2014;5: 10.

24 Roy S, Pantanowitz L, Amin M, Seethala RR, Ishtiaque A, Yousem SA, Parwani AV, Cucoranu I, Hartman DJ: Smartphone adapters for digital photomicrography. J Pathol Inform 2014;5:24. 
25 Speiser JJ, Hughes I, Mehta V, Wojcik EM, Hutchens KA: Mobile teledermatopathology: using a tablet PC as a novel and cost-efficient method to remotely diagnose dermatopathology cases. Am J Dermatopathol 2014;36:5457.

-26 Gavrielides MA, Conway C, O'Flaherty N, Gallas BD, Hewitt SM: Observer performance in the use of digital and optical microscopy for the interpretation of tissue-based biomarkers. Anal Cell Pathol (Amst) 2014;2014:157308.

$\checkmark 27$ Nassar A, Cohen C, Agersborg SS, Zhou W, 3 Lynch KA, Barker EA, Vanderbilt BL, Thompson J, Heyman ER, Olson A, Lange H, Siddiqui MT: A multisite performance study comparing the reading of immunohistochemical slides on a computer monitor with conventional manual microscopy for estrogen and progesterone receptor analysis. Am J Clin Pathol 2011;135:461-467.

28 Micsik T, Kiszler G, Szabo D, Krecsak L, Hegedus C, Tibor K, Molnar B: Computer aided semi-automated evaluation of HER2 immunodetection - a robust solution for supporting the accuracy of anti HER2 therapy. Pathol Oncol Res 2015;21:1005-1011.

29 Krenacs T, Zsakovics I, Diczhazi C, Ficsor L, Varga VS, Molnar B: The potential of digital microscopy in breast pathology. Pathol Oncol Res 2009;15:55-58.

- 30 Al-Janabi S, Huisman A, Vink A, Leguit RJ, Offerhaus GJ, Ten Kate FJ, van Dijk MR, van Diest PJ: Whole slide images for primary diagnostics in dermatopathology: a feasibility study. J Clin Pathol 2012;65:152-158.

- 31 Randell R, Ruddle RA, Mello-Thoms C, Thomas RG, Quirke P, Treanor D: Virtual reality microscope versus conventional microscope regarding time to diagnosis: an experimental study. Histopathology 2013;62:351358.

- 32 Krishnamurthy S, Mathews K, McClure S, Murray M, Gilcrease M, Albarracin C, Spinosa J, Chang B, Ho J, Holt J, Cohen A, Giri D, Garg K, Bassett RL Jr, Liang K: Multi-institutional comparison of whole slide digital imaging and optical microscopy for interpretation of hematoxylin-eosin-stained breast tissue sections. Arch Pathol Lab Med 2013;137: 1733-1739.

- 33 Houghton JP, Ervine AJ, Kenny SL, Kelly PJ, Napier SS, McCluggage WG, Walsh MY, Hamilton PW: Concordance between digital pathology and light microscopy in general surgical pathology: a pilot study of 100 cases. J Clin Pathol 2014;67:1052-1055.

-34 Sanders DS, Grabsch H, Harrison R, Bateman A, Going J, Goldin R, Mapstone N, Novelli M, Walker MM, Jankowski J: Comparing virtual with conventional microscopy for the consensus diagnosis of Barrett's neoplasia in the AspECT Barrett's chemoprevention trial pathology audit. Histopathology 2012;61:795800 .
Randell R, Ruddle RA, Thomas RG, MelloThoms C, Treanor D: Diagnosis of major cancer resection specimens with virtual slides: impact of a novel digital pathology workstation. Hum Pathol 2014;45:2101-2106.

-36 Ordi J, Castillo P, Saco A, Del Pino M, Ordi O, Rodriguez-Carunchio L, Ramirez J: Validation of whole slide imaging in the primary diagnosis of gynaecological pathology in a university hospital. J Clin Pathol 2015;68:3339.

Cornish TC, Swapp RE, Kaplan KJ: Wholeslide imaging: routine pathologic diagnosis. Adv Anat Pathol 2012;19:152-159.

38 Pantanowitz L, Valenstein PN, Evans AJ, Kaplan KJ, Pfeifer JD, Wilbur DC, Collins LC, Colgan TJ: Review of the current state of whole slide imaging in pathology. J Pathol Inform 2011;2:36.

39 Gilbertson JR, Ho J, Anthony L, Jukic DM, Yagi Y, Parwani AV: Primary histologic diagnosis using automated whole slide imaging: a validation study. BMC Clin Pathol 2006;6:4.

40 Jukic DM, Drogowski LM, Martina J, Parwani AV: Clinical examination and validation of primary diagnosis in anatomic pathology using whole slide digital images. Arch Pathol Lab Med 2011;135:372-378.

41 Li X, Liu J, Xu H, Gong E, McNutt MA, Li F, Anderson VM, Gu J: A feasibility study of virtual slides in surgical pathology in China. Hum Pathol 2007:38:1842-1848.

42 Stathonikos N, Veta M, Huisman A, van Diest PJ: Going fully digital: perspective of a Dutch academic pathology lab. J Pathol Inform 2013;4:15.

43 Bauer TW, Schoenfield L, Slaw RJ, Yerian L, Sun Z, Henricks WH: Validation of whole slide imaging for primary diagnosis in surgical pathology. Arch Pathol Lab Med 2013;137: 518-524.

44 Al-Janabi S, Huisman A, Nap M, Clarijs R, van Diest PJ: Whole slide images as a platform for initial diagnostics in histopathology in a medium-sized routine laboratory. J Clin Pathol 2012;65:1107-1111.

45 Pantanowitz L, Wiley CA, Demetris A, Lesniak A, Ahmed I, Cable W, Contis L, Parwani $\mathrm{AV}$ : Experience with multimodality telepathology at the University of Pittsburgh Medical Center. J Pathol Inform 2012;3:45.

46 Al-Janabi S, Huisman A, Willems SM, van Diest PJ: Digital slide images for primary diagnostics in breast pathology: a feasibility study. Hum Pathol 2012;43:2318-2325.

47 Reyes C, Ikpatt OF, Nadji M, Cote RJ: Intraobserver reproducibility of whole slide imaging for the primary diagnosis of breast needle biopsies. J Pathol Inform 2014;5:5.

48 Lopez AM, Graham AR, Barker GP, Richter LC, Krupinski EA, Lian F, Grasso LL, Miller A, Kreykes LN, Henderson JT, Bhattacharyya AK, Weinstein RS: Virtual slide telepathology enables an innovative telehealth rapid breast care clinic. Semin Diagn Pathol 2009;26:177186.
49 Nassar A, Cohen C, Albitar M, Agersborg SS, Zhou W, Lynch KA, Heyman ER, Lange H, Siddiqui MT: Reading immunohistochemical slides on a computer monitor - a multisite performance study using 180 HER2-stained breast carcinomas. Appl Immunohistochem Mol Morphol 2011;19:212-217.

50 Khurana KK: Telecytology and its evolving role in cytopathology. Diagn Cytopathol 2012;40:498-502.

51 Thrall M, Pantanowitz L, Khalbuss W: Telecytology: clinical applications, current challenges, and future benefits. J Pathol Inform 2011;2:51.

52 Donnelly AD, Mukherjee MS, Lyden ER, Bridge JA, Lele SM, Wright N, McGaughey MF, Culberson AM, Horn AJ, Wedel WR, Radio SJ: Optimal z-axis scanning parameters for gynecologic cytology specimens. J Pathol Inform 2013;4:38.

53 Wilbur DC: Digital cytology: current state of the art and prospects for the future. Acta $\mathrm{Cy}$ tol 2011;55:227-238.

-54 Buxbaum JL, Eloubeidi MA, Lane CJ, Varadarajulu $\mathrm{S}$, Linder $\mathrm{A}$, Crowe $\mathrm{AE}$, Jhala $\mathrm{D}$, Jhala NC, Crowe DR, Eltoum IA: Dynamic telecytology compares favorably to rapid onsite evaluation of endoscopic ultrasound fine needle aspirates. Dig Dis Sci 2012;57:3092-3097.

55 Collins BT: Telepathology in cytopathology: challenges and opportunities. Acta Cytol 2013;57:221-232.

$56 \mathrm{Al}$ Habeeb HA, Evans A, Ghazarian D: Virtual microscopy using whole-slide imaging as an enabler for teledermatopathology: a paired consultant validation study. J Pathol Inform 2012;3:2

57 Nielsen PS, Lindebjerg J, Rasmussen J, Starklint H, Waldstrom M, Nielsen B: Virtual microscopy: an evaluation of its validity and diagnostic performance in routine histologic diagnosis of skin tumors. Hum Pathol 2010; 41:1770-1776.

58 Al-Janabi S, Huisman A, Vink A, Leguit RJ, Offerhaus GJ, Ten Kate FJ, van Diest PJ: Whole slide images for primary diagnostics of gastrointestinal tract pathology: a feasibility study. Hum Pathol 2012;43:702-707.

59 Molnar B, Berczi L, Diczhazy C, Tagscherer A, Varga SV, Szende B, Tulassay Z: Digital slide and virtual microscopy based routine and telepathology evaluation of routine gastrointestinal biopsy specimens. J Clin Pathol 2003;56:433-438.

60 Risio M, Bussolati G, Senore C, Vigna S, Frangipane E, Segnan N, Cassoni P: Virtual microscopy for histology quality assurance of screen-detected polyps. J Clin Pathol 2010;63: 916-920.

61 Camparo P, Egevad L, Algaba F, Berney DM, Boccon-Gibod L, Comperat E, Evans AJ, Grobholz R, Kristiansen G, Langner C, Lopez-Beltran A, Montironi R, Oliveira P, Vainer B, Varma M: Utility of whole slide imaging and virtual microscopy in prostate pathology. APMIS 2012;120:298-304. 
62 Helin HO, Lundin ME, Laakso M, Lundin J, Helin HJ, Isola J: Virtual microscopy in prostate histopathology: simultaneous viewing of biopsies stained sequentially with hematoxylin and eosin, and alpha-methylacyl-coenzyme A racemase/p63 immunohistochemistry. J Urol 2006;175:495-499.

63 Chargari C, Comperat E, Magne N, Vedrine L, Houlgatte A, Egevad L, Camparo P: Prostate needle biopsy examination by means of virtual microscopy. Pathol Res Pract 2011; 207:366-369.

64 Fine JL, Grzybicki DM, Silowash R, Ho J, Gilbertson JR, Anthony L, Wilson R, Parwani AV, Bastacky SI, Epstein JI, Jukic DM: Evaluation of whole slide image immunohistochemistry interpretation in challenging prostate needle biopsies. Hum Pathol 2008;39: 564-572.

65 Helin H, Lundin M, Lundin J, Martikainen P, Tammela T, Helin H, van der Kwast T, Isola $\mathrm{J}$ : Web-based virtual microscopy in teaching and standardizing Gleason grading. Hum Pathol 2005;36:381-386.

66 Rodriguez-Urrego PA, Cronin AM, Al-Ahmadie HA, Gopalan A, Tickoo SK, Reuter VE, Fine SW: Interobserver and intraobserver reproducibility in digital and routine microscopic assessment of prostate needle biopsies. Hum Pathol 2011;42:68-74.

-67 Al-Janabi S, Huisman A, Jonges GN, Ten Kate FJ, Goldschmeding R, van Diest PJ: Whole slide images for primary diagnostics of urinary system pathology: a feasibility study. J Renal Inj Prev 2014;3:91-96.

68 Ho J, Parwani AV, Jukic DM, Yagi Y, Anthony L, Gilbertson JR: Use of whole slide imaging in surgical pathology quality assurance: design and pilot validation studies. Hum Pathol 2006;37:322-331.

-69 Sturm B, Fleskens SJ, Bot FJ, van Velthuysen ML, Speel EJ, Slootweg PJ, van der Laak JA: Virtual microscopy is a valid alternative for the diagnostic assessment of laryngeal premalignancies. Histopathology 2014;64:602-604.

70 Wiley CA, Murdoch G, Parwani A, Cudahy T, Wilson D, Payner T, Springer K, Lewis T: Interinstitutional and interstate teleneuropathology. J Pathol Inform 2011;2:21.
Grala B, Markiewicz T, Kozlowski W, Osowski S, Slodkowska J, Papierz W: New automated image analysis method for the assessment of Ki-67 labeling index in meningiomas. Folia Histochem Cytobiol 2009;47:587-592.

72 Alomari YM, Sheikh Abdullah SN, MdZin RR, Omar K: Adaptive localization of focus point regions via random patch probabilistic density from whole-slide, Ki-67-stained brain tumor tissue. Comput Math Methods Med 2015;2015:673658.

73 Al-Janabi S, Huisman A, Nikkels PG, Ten Kate FJ, van Diest PJ: Whole slide images for primary diagnostics of paediatric pathology specimens: a feasibility study. J Clin Pathol 2013;66:218-223.

74 Arnold MA, Chenever E, Baker PB, Boue DR, Fung B, Hammond S, Hendrickson BW, Kahwash SB, Pierson CR, Prasad V, Nicol KK, Barr T: The College of American Pathologists guidelines for whole slide imaging validation are feasible for pediatric pathology: a pediatric pathology practice experience. Pediatr Dev Pathol 2015;18:109-116.

75 Slodkowska J, Chyczewski L, Wojciechowski M: Virtual slides: application in pulmonary pathology consultations. Folia Histochem Cytobiol 2008;46:121-124.

76 Jen KY, Olson JL, Brodsky S, Zhou XJ, Nadasdy T, Laszik ZG: Reliability of whole slide images as a diagnostic modality for renal allograft biopsies. Hum Pathol 2013;44:888894.

77 Furness P: A randomized controlled trial of the diagnostic accuracy of internet-based telepathology compared with conventional microscopy. Histopathology 2007;50:266-273.

78 Baak JP, van Diest PJ, Meijer GA: Experience with a dynamic inexpensive video-conferencing system for frozen section telepathology. Anal Cell Pathol 2000;21:169-175.

79 Ramey J, Fung KM, Hassell LA: Use of mobile high-resolution device for remote frozen section evaluation of whole slide images. J Pathol Inform 2011;2:41.
80 Buck TP, Dilorio R, Havrilla L, O’Neill DG: Validation of a whole slide imaging system for primary diagnosis in surgical pathology: a community hospital experience. J Pathol Inform 2014;5:43.

81 Campbell WS, Lele SM, West WW, Lazenby AJ, Smith LM, Hinrichs SH: Concordance between whole-slide imaging and light microscopy for routine surgical pathology. Hum Pathol 2012;43:1739-1744.

82 Dangott B, Parwani A: Whole slide imaging for teleconsultation and clinical use. J Pathol Inform 2010;1:7.

83 Song Y, Treanor D, Bulpitt AJ, Magee DR: 3D reconstruction of multiple stained histology images. J Pathol Inform 2013;4:S7.

84 Caie PD, Turnbull AK, Farrington SM, Oniscu A, Harrison DJ: Quantification of tumour budding, lymphatic vessel density and invasion through image analysis in colorectal cancer. J Transl Med 2014;12:156.

85 Murakami Y, Abe T, Hashiguchi A, Yamaguchi M, Saito A, Sakamoto M: Color correction for automatic fibrosis quantification in liver biopsy specimens. J Pathol Inform 2013;4:36.

86 Neil DA, Roberts IS, Bellamy CO, Wigmore SJ, Neuberger JM: Improved access to histopathology using a digital system could increase the organ donor pool and improve allocation. Transpl Int 2014;27:759-764.

87 Neltner JH, Abner EL, Schmitt FA, Denison SK, Anderson S, Patel E, Nelson PT: Digital pathology and image analysis for robust highthroughput quantitative assessment of $\mathrm{Alz}$ heimer disease neuropathologic changes. J Neuropathol Exp Neurol 2012;71:1075-1085.

88 Riber-Hansen R, Vainer B, Steiniche T: Digital image analysis: a review of reproducibility, stability and basic requirements for optimal results. APMIS 2012;120:276-289.

89 Webster JD, Michalowski AM, Dwyer JE, Corps KN, Wei BR, Juopperi T, Hoover SB, Simpson RM: Investigation into diagnostic agreement using automated computer-assisted histopathology pattern recognition image analysis. J Pathol Inform 2012;3:18.

90 Webster JD, Dunstan RW: Whole-slide imaging and automated image analysis: considerations and opportunities in the practice of pathology. Vet Pathol 2014;51:211-223. 